\title{
Occasional Observation of Acanthamoeba in the Pharynx. A Case Report
}

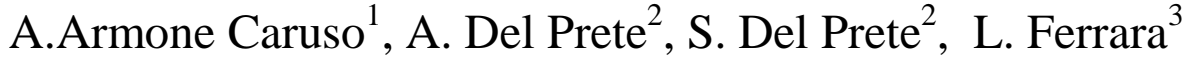 \\ ${ }^{1}$ ENT Department, AIAS Structure of Afragola, Contrada Leutrec Snc, 80021 Naples, Italy. \\ ${ }^{3}$ Department Of Head And Neck. University of Naples Federico II, Via Pansini 5, 80131 Naples, Italy \\ ${ }^{3}$ Department Of Pharmacy,University of Naples Federico II, Via Domenico Montesano 49, 80131 Naples, Italy
}

\begin{abstract}
Acanthamoeba is an opportunistic protozoan "free-living" widely distributed in nature and is a potential pathogen for Man. It is responsible for pathologies inherent in the CNS, the cornea, the skin, especially in immunocompromised subjects. An adult subject of 70 years old, suffering from acute pharyngitis, was subjected to a cytological sampling of the pharyngeal mucosa by means of the modified Ayre spatula type A from Armone Caruso, to perform either a coloring method according to the May-Grunwald - Giemsa, and observed under the microscope optical with oil immersion at $100 x$ of fifty fields, that a preparation for observation under the SEM.( Scanning Electron Microscope,). In both observations it was highlighted in the form of trophozoite some rare Acanthamoeba. The subject was observed without that have appeared due to protozoan diseases: in practice it was a casual observation. The clinical case, described occasionally, it shows how the distribution in the environment of these amoebae is very extensive and mainly related to water and become of interest about the health, for the human use of the latter for purposes such as hygiene care, bathing, medical and health applications. The objective of this paper is to provide a starting point to discuss the potential pathogenicity associated with the presence of Acanthamoeba in the water ecosystems. This is for raise the awareness the scientific community and promote environmental control initiatives of this pathogen in both outdoor and indoor environments.
\end{abstract}

Keywords: Acanthamoeba, Pharynx, Cytological Evaluation, Optical Microscopy, Scanning Electron Microscope

\section{Introduction}

The Acanthamoeba is an opportunistic protozoan widely distributed in nature. Its existence is constituted by two stages of life: an active, trophozoite , during which the protozoan reproduces; the other, quiescence, cystic form that can last several years. The cysts measure how a red blood cell from 5 to $10 \square$. The trophozoites are from 40 to $50 \square$, but may increase in a suitable environment up to $200-400 \square \square$

During the last decades, studies on the Acanthamoeba were encouraged because they are highlighted a progressive keratitis and encephalitis caused by this protozoan "free-living." In fact, since 1970 it has been identified as a cause of human diseases and has been isolated in soil, water, air and dust [1-3].

As part of the many species of protozoa "free-living", the species belonging to the genera the Acanthamoeba, Naegleria and Balamuthia spp. [Table 1] are able to parasitize humans and become pathogenic causing even fulminant infections of the central nervous system. Naegleria species differ in the pathogenesis from the others because they determine the primary amoebic meningoencephalitis ( known as PAM) as the infection due to the other two amoebas "free-living" is granulomatous amoebic encephalitis (known as GAE). In some cases of immunodeficiency it may be found in rhinitic forms and sinusitis [4-6]. These encephalic infections initially show subacute symptoms with a fast subsequent course with induction of pro-inflammatory response that quickly leads the tissue necrosis and inevitably fatal outcome. This pathogenesis is rare and typical of immune-compromised patients (HIV +, immune -suppressed, alcoholist) but the Acanthamoeba also determines an ocular disease in immune-competent subjects, especially in carriers of contact lenses, especially with those soft [7] with serious infection of the cornea (keratitis amoebic ) that, if neglected, leading to blindness. The Acanthamoeba was described for the first time in 1930 [8] by Castellani in Cryptococcus pararoseus crops. Recently the Acanthamoeba was also associated with skin lesions in patients with AIDS, as well as in pneumonia (especially as vehicle of Legionella) but, as already said, the ocular pathology (amoebic keratitis) which determines is in load of healthy people and immune-competent subjects. The factors responsible for the pathogenic free-living amoebae are still poorly understood, but the pathogenesis of generating is probably due to the inflammation that triggers in the host [9]. It was however demonstrated for these amoebae an ability to cause cytopathic effect in vitro on cell cultures [10] and it seems that the degree of pathogenicity is in close correlation with their thermo-tolerance. From the sixties began the first observations on the pathogenic role of Acanthamoeba in some experimentally infected mice, after being found as a pollutant of cell cultures maintained in the laboratory. Since then they began the first comments on the pathogenic role of Acanthamoeba 
in the studies of two Australian clinicians that pointed out with histological techniques these protozoa in brain of two people died of meningoencephalitis [11]. They were then reported in several countries in the world, more and more cases of infection attributable to free-living amoebae. In particular, some species of Acanthamoeba were generally recognized as etiologic agents of a form of chronic and progressively destructive keratitis [12].

Laboratory diagnosis is based primarily on identifying direct microscopy, optical microscopy and / or SEM, or with in vivo confocal microscope (ConfoScan Nidek) and / or cultivation of cysts from "scraping" Corneal, biopsies, contact lenses and liquid conservation of the lenses. Recently the methods of PCR (Polymerase Chain Reaction) have been used for the identification of the Acanthamoeba spp., useful for a rapid and sensitive diagnosis of human cases of keratitis and for the identification of the genotype. As for Italy little is known about the epidemiology of amebiasis in humans and the presence of parasites in the environment, the genotypes present and possible routes of transmission: the number of confirmed cases of infection due to this protozoan could be an underestimate

\section{Case Report and Identification Method}

A patient 70 years old, male, suffering from acute pharyngitis has arrived at the 'Clinic of ENT and nasal cytology of' AIAS (Italian Association of Assistance Disadvantaged) Afragola. For the study of this pathology the patient has been subjected to an exfoliative semiquantitative evaluation of the rhinopharynx, as viewed by optical microscope and SEM. After the completion of the procedures on privacy and informed consent, we have performed an objective examination of the oral cavity, using a headlamp WIDA 30850 fiber optic (Gima - Milan) and a disposable plastic spatula (Gima - Milano). Subsequently, we have performed an double levying exfoliative cytology, in the back of the throat region, using two disposable Ayre spatulas type A modified by Armone Caruso [Fig. 1] to perform a cytological study by light microscopy and SEM.

The cells for be analyzed with optical microscope were placed on a glass slide cytology ,electrostatically charged(, SuperFrost ${ }^{\circledR}$ Plus Menzel - Gläser Thermo Scientific). We proceeded to staining in solution, according to the method panoptic Pappenheim (3 min. in the May-Grunwald dye pure; 6 min. in MayGrunwald to $50 \% ; 1 \mathrm{~min}$. in distilled water; $30 \mathrm{~min}$. in Giemsa 1:10). The slide was then covered with a cover glass with dimensions of 24 x $50 \mathrm{~mm} \# 1$ and observed under an optical microscope Nikon Eclipse 200, to $100 \mathrm{x}$ magnification in oil-immersion. For the acquisition of microscope images has been used a Nikon DS FI1 camera with a program of acquisitions images NIS - Elements D Version 2.30. For the semi-quantitative evaluation is a suitable table was used [13].

For the examination with SEM (Scanning Electron Microscope), the samples of epithelium resting on slides with a diameter of $1 \mathrm{~cm}$ were fixed in $3 \%$ glutaraldehyde in a $0.065 \mathrm{M}$ phosphate buffer(pH 7.4 ) for $2 \mathrm{~h}$ at room temperature. Slides were washed three times in $0.065 \mathrm{M}$ phosphate buffer (for $30 \mathrm{~min}$.), then placed in $1 \%$ OsO4 in 0.064M phosphate buffer ( $\mathrm{pH} 7.4$ )for $30 \mathrm{~min}$. Samples were dehydrated through a graded series of ethanol, and then dried in critical point- CO2 liquid Bemar SPC 1500 apparatus (Bomar Co., Tacome, WA, USA). Specimens were mounted on aluminum stubs with silver-conducting paint, sputtered with a thin $(20 \mathrm{~nm})$ films of gold and observed with a Cambridge Mark 250 SEM [14].

In both observations, we noted a situation compatible with a cellular irritation framework. You were detected in both the microscopic examinations, an Acanthamoeba colony during trophozoite and cystic form [Fig.2, 3 SEM]. The subject has been subjected to local treatment with Chlorhexidine $0.2 \%$ three times a day for ten days.

As a result of this observation, all frequent clinical investigations were carried out to analyze the state of health of the subject (eye exams, ent and infectious visits) subjecting it to appropriate therapy. Furthermore it proceeded to a sixty-day observational study to evaluate a possible worsening of the welfare state. Also it was negative. After this period has been observed once again and there has been a clinical recovery.

\section{Results and Discussion}

Acanthamoeba is a protozoan that can be frequently observed in the fresh samples without refrigerate and in any case within 24 hours [15]. The diagnostic protocol to the microscope may involves the assembly of a slide fitted with the "scraping" levy. The coloring is done with the classic colorimetric methods such as Giemsa and PAS (Periodic Acid-Schiff). It is possible also color the histological sections with hematoxylin-eosin and PAS. With the fluorescence microscope you can show cysts and trophozoites after staining the sample with chrome fluoride (Calcofluor white). Acanthamoeba cysts take the apple-green fluorescence, while the other cells present, including any trophozoites turn red-brown. A non-invasive method used in ophthalmology in recent years is the confocal microscopy which allows it to put in evidence cysts and trophozoites in the patient's corneal tissue as hyper-reflective structures [16].

The examination requires, for people suffering from keratitis, a good patient compliance and, often, the intense photophobia and pain it can make problematic the execution. The pathology laboratory diagnosis 
Acanthamoeba is based on identifying microscopic direct and / or culture of the cysts and trophozoites after withdrawal by trowel of the surface layer of the cornea.

The subject observed by us has shown a form of acute pharyngitis; at the light of microscopic observations could be secondary to the protozoan same. This eventuality could be connected also to the disappearance of the symptoms after appropriate therapy, with Chlorhexidine $0.2 \%$ for local use, three times daily for ten days.

As a result of this observation, all frequent clinical investigations were carried out to analyze the state of health of the subject (eye exams, ent and infectious visits) subjecting it to appropriate therapy. Furthermore it proceeded to a sixty-day observational study to evaluate a possible worsening of the welfare state. Also it was negative. After this period has been observed once again and there has been a clinical recovery.

Acanthamoeba is a protozoan that can be frequently observed in the fresh samples without refrigerate and in any case within 24 hours [17]. The diagnostic protocol to the microscope may involves the assembly of a slide fitted with the "scraping" levy. The coloring is done with the classic colorimetric methods such as Giemsa and PAS (Periodic Acid-Schiff). It is possible also color the histological sections with hematoxylin-eosin and PAS. With the fluorescence microscope you can show cysts and trophozoites after staining the sample with chrome fluoride (Calcofluor white). Acanthamoeba cysts take the apple-green fluorescence, while the other cells present, including any trophozoites turn red-brown. A non-invasive method used in ophthalmology in recent years is the confocal microscopy which allows it to put in evidence cysts and trophozoites in the patient's corneal tissue as hyper-reflective structures [18].

The examination requires, for people suffering from keratitis, a good patient compliance and, often, the intense photophobia and pain it can make problematic the execution. The pathology laboratory diagnosis Acanthamoeba is based on identifying microscopic direct and / or culture of the cysts and trophozoites after withdrawal by trowel of the surface layer of the cornea.

The subject observed by us has shown a form of acute pharyngitis; at the light of microscopic observations could be secondary to the protozoan same. This eventuality could be connected also to the disappearance of the symptomatology after appropriate therapy, with Chlorhexidine $0.2 \%$ for local use, three times daily for ten days.

The identification of Acanthamoeba, which in this case could be responsible for acute pharyngitis, can open a debate to raise awareness of the scientific community and to support environmental control initiatives of this pathogen in both outdoor and indoor environments. In fact, the transmission depends primarily on two factors: the virulence of the parasite and the immune status of the subject. The survival of these free-living amoebae is linked to aquatic ecosystems and consensus is that, in doing so, can easily reach the waters of common use for the population and to make contact with the man. Even the presence of trophozoites and cysts of Acanthamoeba in soil is correlated with the presence in the waters due to the run-off, and the course of the water, not to mention the possible dispersal of cysts, particularly light, into the air.The amoebae, in general, feed on bacteria and for the Acanthamoeba, in particular, has been widely demonstrated the ability to convey, in this way, the pathogenic species such as Legionella, of great medical importance to humans. The bacterial and viral species for which is gradually demonstrating the vector capacity by Acanthamoeba are, in reality, many and with a diversified pathogenicity. The other vehicular potential pathogenic protozoa due to "water-borne" infections has yet to be assessed.

Even in the case of special health interest for the aquatic viruses, such as adenovirus [17,18], it would be appropriate to evaluate the implication. It is therefore inevitable to point out that you should not underestimate the health problem caused or induced by this free-living amoeba whose pathogenic role is proving emerging. It is also clear that a diagnostic procedure should provide the molecular specific characterization in diagnosing of each case, the species, the infecting genotype and its possible pathogenicity, since not all species and genotypes are pathogenic.

It remains, therefore, a high health interest, concerning the presence of Acanthamoeba in the waters for anthropic use, particularly for bathing and for medical purposes.

It should, in fact, be remembered that the contamination of water for potable use by this protozoan does not represent a health problem connected with the ingestion, but only with the contact, as happens when the tap water is used for purposes other than drinking or bathing.

\section{Conclusion}

The presence of Acanthamoeba should be considered a potential threat to the health associated with human activities in catchment basins of surface, in particular for the contact with water, and then for bathing. Its presence in surface water should be seriously considered as a potential threat to public health if its waters are used for bathing activities..

Its presence is still described in air conditioning equipment, and in air conditioners for both cooling water system to which air due to the volatility of the cysts, very slight and then both incoming and outgoing. To 
date has not yet been well investigated its implication in nosocomial infections, although there are some evidences regarding Legionella, at whose is closely associated, and has not been evaluated, as with other protozoa, what is the containment degree exerted by the current disinfection protocols. It is therefore essential to deepen the knowledges, in order to propose guidelines for monitoring the aquatic ecosystems, for to control of this pest and prevent harmful exposure of the population through the design of any specific devices to avoid the infections [10].

\section{Figures And Tables}

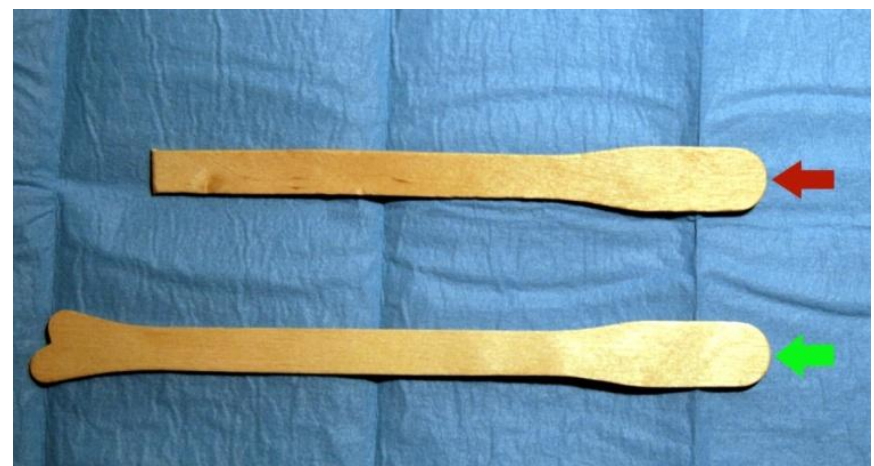

FIG. 1 : Ayre spatula type A, green arrow; Ayre spatula type A modified, red arrow. For pharyngeal scraping it was used the convex side indicated by the arrow.

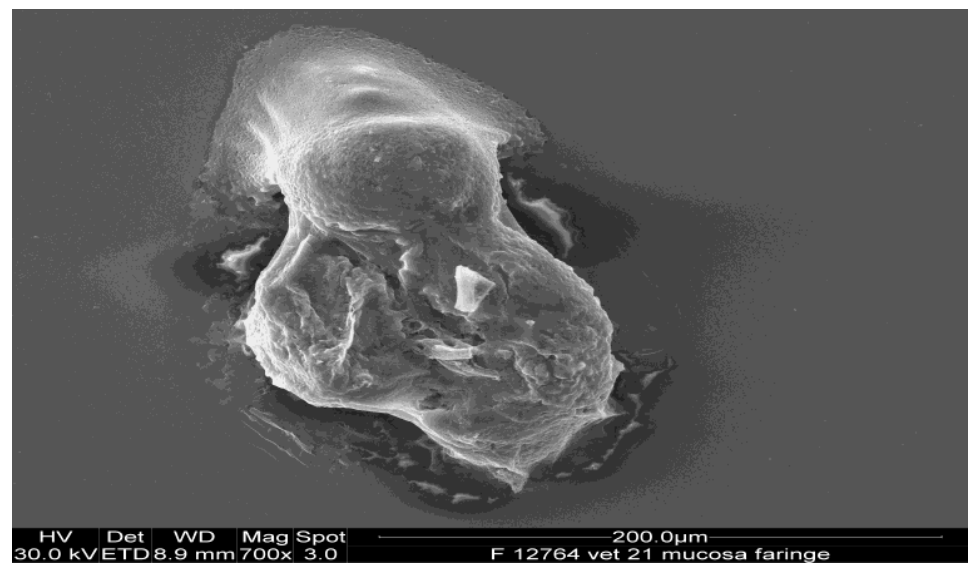

FIG. 2 SEM microscopy of the scraping pharyngeal cells, magnification $200 \square$. Acanthamoeba is visible in the form of trophozoite

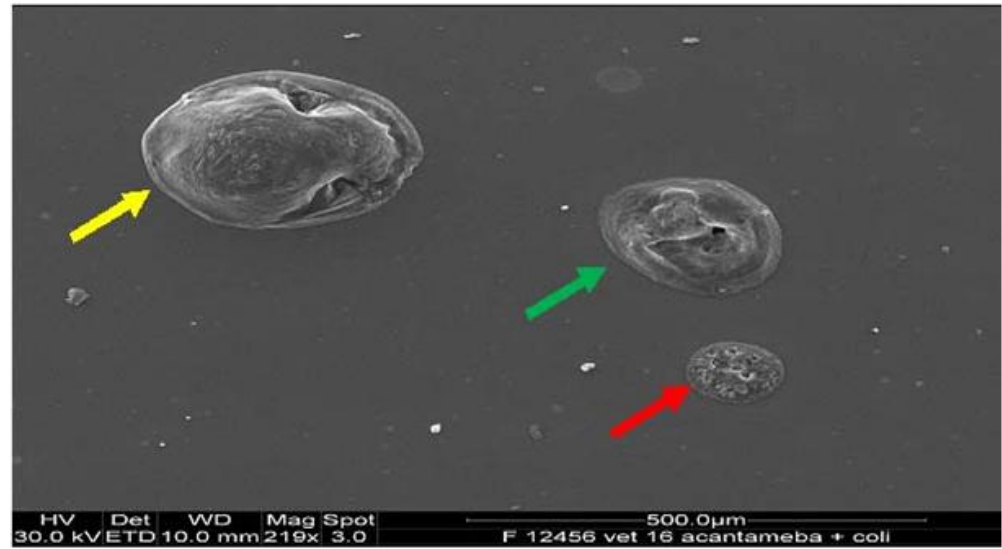

FIG. 3 SEM microscopy of the scraping pharyngeal cells, magnification 500 microns. Acanthamoeba observed in various stages: red arrow, cystoid phase; green arrow, trophozoite; yellow arrow, trophozoite observed from the upper pole 
TAB.1 Phylogenetic scheme of Acanthamoeba, Balamuthia, and Naegleria. From F.Marciano-Cabral, G. Cabral Acanthamoeba spp. as Agents of Disease in Humans. Clinical Microbiology Reviews. 2003;16(2):273307.

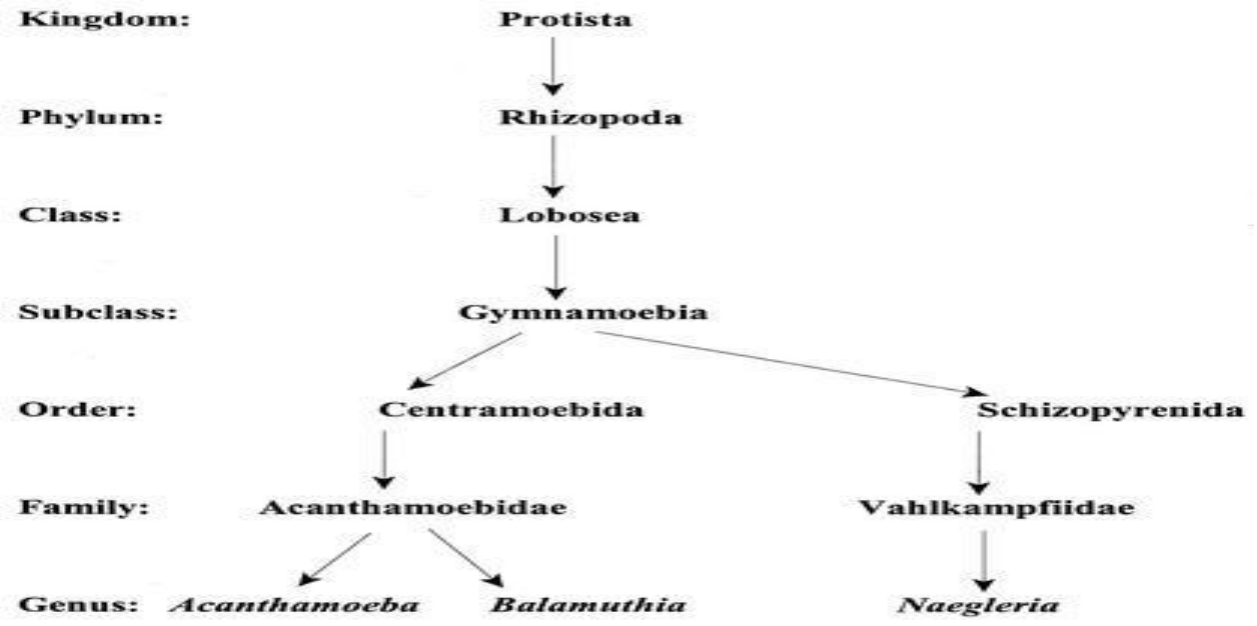

References

[1] R.Siddiqui, A.Khan, Biology and pathogenesis of amoeba. Parasites Vectors. 5, 2012,1-6. doi: 10.1186/1756-3305

[2] S.Klivington, DG. White, Acanthamoeba: biology, ecology and human disease. ClinMicrobiol Rev. 5,1994,12- 20.

[3] PI.Gunawan, A. Idarto, D. Saharso, Acanthamoeba Infection in a Drowning Child, Ethiop J Health Sci.26(3), 2016 ,289-92

[4] ] VA. Dunand, SM. Hammer, R. Rossi,M.Poulin,MA.Albrecht,JP Doweiko,PC.De Girolami, E.Coakley, E.Piessens,CA.Wanke, Parasitic Sinusitis and Otitis in Patients Infected with Human Immunodeficiency Virus: Report of Five Cases and Review. Clinical Infectious Diseases 25,1997,267-72

[5] EW.Godofsky, J. Zinreich, M.Armstrong, JM.Leslie, CS Weikel, Sinusitis in HIV-infected patients: a clinical and radiographic review. Am J Med 93,1992, 163-70

[6] L.Cugino , J.Butcher, WL.Hoppes, M.Doyle, C.Bogden, Acanthamoeba sinusitis in a patient with AIDS [abstract no. 451]. Clin Infect Dis , 21,1995,795

[7] R. Forte, G. Cennamo, S. Del Prete, I. Cesarano, and A. Del Prete, Scanning Electron Microscopy of Corneal Epithelium in Soft Contact Lens Wearers. Cornea 29, 2010, 732-736

[8] A.Castellani, Furunculosis Cryptococcica vel Blastomycetica vel Moniliaca: (Folliculitis decalvans cryptococcica pro parte). Proc $R$ Soc Med 23(7), 1930; 1042-3.

[9] NA. Khan, Novel in vitro and in vivo models to study central nervous system infections due to Acanthamoeba spp. Exp Parasitol 126, 2009,69-72.

[10] R. Siddiqui, NA.Khan, Biology and pathogenesis of Acanthamoeba. Parasit Vectors 5,6, 2012,doi:1186/1756- 3305

[11] CG..Culbertson, JW Smith, JR Minner, Acanthamoeba: osservazioni su animali di patogenicità. Scienza127 (3313), $1958,1506$.

[12] M. Fowler, RF.Carter, Acute pyogenic meningitis probably due to Acanthamoeba sp.: a preliminary report. Br Med $J$ 2(5464), 1965,740-2.

[13] J.Naginton, PG.Watson,TJ. Playfair, J.McGill, BR.Jones, AD.Steele, Amoebic infection of the eye. Lancet 2 (7896), 1974,1537-40.

[14] L. Ferrara, D. Naviglio, A.Armone Caruso, Approach under the form of semiquantitative cytological evaluation for chronic pharyngitis, Eurasian Multidisciplinary Forum, 24-26 October, Tbilisi, Georgia, Proceedings, ESI journal special edition Vol.3, 2013, 218-221

[15] GL. Cennamo, A. Del Prete, R. Forte, G. Cafiero, S. Del Prete and D. Marasco Impression cytology with scanning electron microscopy: a new method in the study of conjunctival microvilli Eye, 22, 2008, 138-143

[16] M.C. Angelici, A. De Sanctis, E. Funari, D. Di Cave, F. Mantelli, S., Acanthamoeba nelle acque: un problema di sanità pubblica in Italia. Rapporti ISTISAN 13/16,2013,Roma 